\title{
Towards single molecule analysis in PDMS microdevices: from the detection of ultra low dye concentrations to single DNA molecule studies
}

\author{
A. Ros*, W. Hellmich, T. Duong, D. Anselmetti \\ Experimental Biophysics, Physics Faculty, Bielefeld University, Universitätsstr. 25, 33615 Bielefeld, Germany
}

Received 15 December 2003; received in revised form 30 March 2004; accepted 1 April 2004

\begin{abstract}
In this paper, we report on the performance of electrophoretical separation and laser-induced fluorescence (LIF) detection of dyes and fluorescently labeled biomolecules in poly(dimethylsiloxane) (PDMS) microdevices. The dyes fluorescein and fluorescein isothiocyanate (FITC) have been separated effectively in $\mathrm{nM}$ concentrations. Fluorescein injections gave linear concentration response in the range from 4 to $100 \mathrm{pM}$. As ultimate detection sensitivity, $100 \mathrm{fM}$ injected fluorescein was obtained. Further, $100 \mathrm{fM}$ injected fluorescein could be detected. This is to our knowledge the smallest electrokinetically injected dye concentration detected on a microchip. Injection studies of fluorescently labeled avidin revealed a theoretical detection limit of $25 \mathrm{nM}$ for laser-induced fluorescence detection in good agreement with separations in glass chips. Furthermore, the injection of several and even one single DNA molecule using a PDMS cross injector has been demonstrated as well as free solution separation of $\lambda$ - and T2-DNA (60 pM each) in periodically structured channels.
\end{abstract}

Keywords: Poly(dimethylsiloxane); Single molecule detection; Microchip separation; DNA migration

\section{Introduction}

Over the recent years, poly(dimethylsiloxane) (PDMS) has been intensively used for the fabrication of microfluidic devices. PDMS allows the so called rapid prototyping (Duffy et al., 1998), in which microstructures can be fabricated with low cost photomasks with channel widths $>20 \mu \mathrm{m}$ and within the timescale of one day with standard photolithogra-

\footnotetext{
* Corresponding author. Fax: +49-521-1065-403.

E-mail address: alexandra.ros@physik.uni-bielefeld.de
} (A. Ros). phy techniques. Additionally, molding of the viscose pre-polymer allows PDMS device fabrication outside a clean room (but under laminar flow benches) and opens the way for applications in a manifold of research areas. Furthermore, high aspect ratios and $\mathrm{nm}$ sized feature sizes can be achieved (Delamarche et al., 1997; Xia and Whitesides, 1998). PDMS is gas permeable and also non-toxic to cells and proteins (Ng et al., 2002). It is therefore not astonishing that PDMS devices have also been used for biomolecule analysis on microchips.

Examples for biomolecule analysis on PDMS comprise several types of on-chip capillary electrophesis 
(CE) separations. Fluorescently labeled amino acids and hydrophobic peptides have been separated by microchip CE (Lacher et al., 2003). Separation of a carbonic anhydrase, as well as an insulin charge ladder, has been reported on PDMS microchips with laser-induced fluorescence (LIF) detection (Duffy et al., 1998). An integrated fluorescence detection system was proposed for the detection of labeled marker proteins by Chabinyc et al. (2001). One-dimensional (Slentz et al., 2001) and three-dimensional (Slentz et al., 2003) electrochromatography of a fluorescein isothiocyanate (FITC)-labeled tryptic digest of BSA have also been demonstrated in PDMS microchips. Applications to DNA include restriction fragment analysis (Effenhauser et al., 1997), as well as free solution DNA migration studies (Duong et al., 2003; Inatomi et al., 2003).

The silicone elastomer PDMS is chemically related to glass, so that microchip capillary electrophoresis (CE) characteristics should be comparable to standard chip analysis in glass. However, PDMS devices often exhibit lower separation efficiencies and lower detection sensitivity compared to conventional glass chips as observed experimentally (Ocvirk et al., 2000; Lacher et al., 2003). In the latter, Liang et al. (1996) demonstrated a detection limit of $3 \mathrm{nM}$ fluorescein isothiocyanate, whereas detection limits in the $\mathrm{pM}$ range have been reported for injected fluorescein (Chiem and Harrison, 1997; Ocvirk et al., 1998) and Cy-5 (Jiang et al., 2001) with LIF detection. In contrast to glass chips, Chabinyc et al. (2001) report a detection limit of $25 \mathrm{nM}$ fluorescein in PDMS with an integrated micro avalanche photodiode detector for fluorescence detection. Glass microchips were further applied for the separation of fluorescently labeled proteins. Applications comprised the separation of labeled marker proteins with $\mu \mathrm{M}$ concentrations and LIF detection (Liu et al., 2000a), an assay for theophylline in the therapeutically useful range (Chiem and Harrison, 1997), as well as a postcolumn labeling method with a mass detection limit of $<0.5 \mathrm{pg}$ for model proteins (Liu et al., 2000b).

To profit from the advantages of PDMS replica molding it is important to achieve sensitive and efficient biomolecule detection and separation in PDMS devices comparable to other microchip materials, especially such as glass chips. Thus, in this paper, we will present the detection of ultra low concentrations of fluorescent dyes in PDMS devices down to $100 \mathrm{fM}$. Additionally, an example of protein microchip CE analysis and single molecule migration studies of DNA will be demonstrated. The latter include the injection of single DNA molecules and the separation of minute DNA samples in periodically structured PDMS channels.

\section{Experimental}

\subsection{Chemicals and reagents}

PDMS (Sylgard 184) was purchased from Dow Corning (USA). Glass slides and cover slides $(76 \mathrm{~mm}$ $\times 26 \mathrm{~mm}$ ) were from Menzel (Germany). Fluorescein isothiocyanate, fluorescein and avidin were obtained from Sigma-Aldrich (Germany). Bovine serum albumine (BSA), $\mathrm{NaCl}, \beta$-mercaptoethanol, T2-DNA (164 kbp), $\lambda$-DNA (48.5 kbp) and tris(hydroxymethyl)aminomethane (TRIS) were purchased from Fluka (Germany). SU-8 50 negative photoresist, thinner $\gamma$-butyrol acetone and developer propyleneglycolmethylether acetate were obtained from Microresist (Germany). Si-wafer (P-Type 100, doped with boron) were from CrysTec (Germany). Tridecafluoro-1,1,2,2tetrahdyrooctyl-1-trichlorsilane (TTTS) was from ABCR (Germany) and YOYO-1 was obtained from Molecular Probes. For all solutions deionized water from a Milli-Q biocel (Millipore, USA) was used.

\subsection{Fabrication of PDMS devices}

The fabrication of the microchip can be divided into two parts: (i) fabrication of a masterwafer with the desired microstructures for multiple PDMS replica castings and (ii) the production of the PDMS replica itself. The production steps for the masterwafer with the inverted structures was recently published (Duong et al., 2003). Briefly, a Si-wafer was spincoated with a negative photoresist SU-8, UV-exposed through a chromium mask and developed in a developer bath. Additional silanization of the masterwafer in a vacuum exsiccator for $30 \mathrm{~min}$ with TTTS enabled the production of many PDMS replicas from the same wafer.

A mixture of Sylgard 184 and its curing agent in a ratio of 10:1 was poured over the wafer. After curing at a temperature of $85^{\circ} \mathrm{C}$ for $4 \mathrm{~h}$ the cross-linked 
polymer was easily peeled off the wafer and reservoir holes were punched through the structured side. The PDMS slab and a clean glass slide were oxidized in an UVO cleaner (Model 42-220, Jelight, USA) for $3 \mathrm{~min}$. After the oxygen treatment, PDMS slab was placed onto the glass slide forming an irreversible seal. A microchannel with periodical structuring of $3 \mu \mathrm{m}$ and a depth and width of 3 and $2.7 \mu \mathrm{m}$, respectively, linked to a cross injector was used for the DNA separation experiment (see inset of Fig. 6 and Duong et al., 2003). For injections of dyes and avidin, a masterwafer containing a cross injector and linear channels with a width of $50 \mu \mathrm{m}$ and a depth of $6.5 \mu \mathrm{m}$ was used.

\subsection{Fluorescence detection}

The experimental setup was mounted on an inverted light microscope (Axiovert 100, Zeiss, Germany), which additionally served as a platform for the confocal laser-induced fluorescence detection system. Via two mirrors (New Focus, USA) the wavelength of an $\mathrm{Ar}^{+}$-laser (Spectra Physics $(5 \mathrm{~mW}$ ) or Omnichrome (max. $25 \mathrm{~mW}$, adjustable), Germany) was coupled into the rear port of the microscope. The excitation light was reflected by a dichroic mirror and focused by a $20 \times$ objective (Zeiss, Germany) into the microchannel. The detection window was adjusted with the $x-y$-stage along the separation channel in various distances from the injector (usually several $\mathrm{mm}$ ). The emitted fluorescence light was collected by the objective and passed through the dichroic mirror and a longpass filter $(520 \mathrm{~nm})$. The tube lens focused the emission light through a $x / y$ adjustable $200 \mu \mathrm{m}$ wide pinhole onto the photomultiplier (Hamamatsu H6240, Japan).

An analogue setup was used for the detection of individual migrating DNA molecules in the structured microchannels. Instead of the confocal laser setup, a mercury arc lamp (HBO 50, Zeiss) was used for excitation. An optimized fluorescence filter set for the dye YOYO-1 (XF100-3, Omega, USA), a 100× oil immersion objective (Plan Neofluar NA 1.3, Zeiss) and a sensitive interline transfer CCD-camera (Imager 3LS, LaVision, Germany) replaced the corresponding parts (for more details see (Duong et al., 2003)).

\subsection{Chip operations}

Microchannels serving for the protein separation experiments were primarily filled with a $50 \mathrm{mg} / \mathrm{mL}$ solution of BSA in $10 \mathrm{mM}$ TRIS buffer and washed thoroughly before injection experiments. For all injections, $10 \mathrm{mM}$ TRIS buffer at $\mathrm{pH} 8.3$ was used. Additionally, $100 \mathrm{mM} \mathrm{NaCl}, 2 \%$ (v/v) $\beta$-mercaptoethanol and YOYO-1 in a ratio of 1:7.5 (YOYO-1:DNA bp) were added to the TRIS buffer for DNA migration experiments. For both experiments, the corresponding buffer was placed into one reservoir. By applying vacuum to the other reservoirs, the microchannels were filled with the buffer solution. Four platinum electrodes were dipped into the reservoirs providing the electronic connection to the microchip. Voltage a) floating injection phase

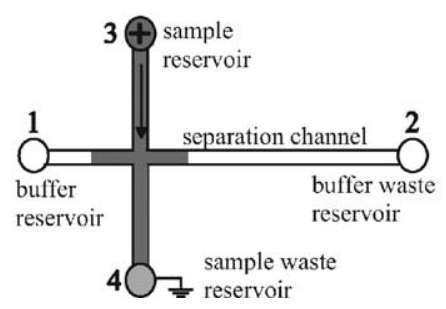

\section{b) pinched injection phase}

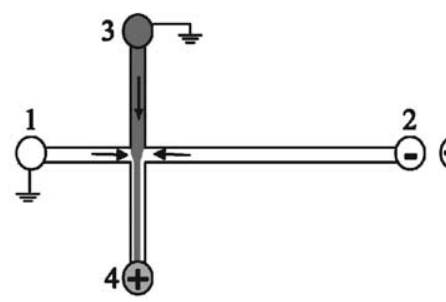

c) separation phase

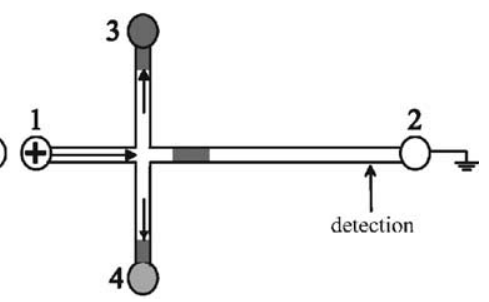

Fig. 1. A schematical view of the injection modi used in this work. (a) In the loading phase of the floating injection, the sample solution is pumped from the sample reservoir to the sample waste reservoir. Analyte leakage into the separation channel is confined through back voltages at reservoirs 1 and 2. (b) During the loading phase of the pinched injection, additional positive voltages are applied at buffer and buffer waste reservoir to avoid leakage into channels 1 and 2. A typical V-shape of analyte flow is established. (c) The sample plug is dispensed into the separation channel by applying a positive voltage to the buffer reservoir and grounding the sample waste reservoir. Exemplarily, the separation phase for the floating injection is presented. The sample plug length varies according to the applied method in the loading phase. With the pinched injection, smaller more defined sample plugs are created compared to the floating injection. 
was applied using power supplies from FUG (Modell HCN 14-12500, HCN 7E-12500 and HCL 14-12500), Germany. Instrumental control and data acquisition were performed with software written in LabView (National Instruments, USA).

The floating method (Zhang and Manz, 2001) was used for protein and dye injections. In the injection phase, a positive voltage at the sample reservoir loaded the analyte into the cross injector of the microstructure. In the separation phase the analyte was dispensed into the separation channel by applying a positive voltage to the buffer reservoir. All other reservoirs were grounded (see Fig. 1a and c).

For injection of DNA into the cross injector, the pinched method (Zhang and Manz, 2001) was used. In the loading phase, channels 1 and 3 were grounded, and -210 and $+150 \mathrm{~V}$ were applied at reservoirs 2 and 4, respectively. For injection of several molecules into the separation channel from a sample containing $60 \mathrm{pM} \lambda$ - and T2-DNA each, $-200 \mathrm{~V}$, GND, $+225 \mathrm{~V}$ and $+125 \mathrm{~V}$ were applied at channels $1-4$, respectively. One single DNA in a solution of $6 \mathrm{pM}$ was injected using lower injection voltages of +150 and $+75 \mathrm{~V}$ at channels 3 and 4 , respectively.

Scanning electron micrographs of PDMS microchannels were performed with a JSM-880 (Jeol, Japan) at an acceleration voltage of $4 \mathrm{kV}$. Prior to imaging a $20 \mathrm{~nm}$ thick gold layer was evaporated (MCS 10, Bal-Tec, Lichtenstein) on the PDMS microchannels. The channel height of the masterwafer was measured by profilometry (Dektak3030ST, Veeco, USA).

\section{Results and discussion}

\subsection{Fluorescent dye separation and detection}

The fabrication method of PDMS devices has been thoroughly described in a recent publication (Duong et al., 2003). Analytes were detected by laser-induced fluorescence detection mounted on an inverted microscope, serving as a robust tool for microchip handling and detection optics. In order to determine the sensitivity of the complete setup fluorescent dyes have been intensively studied. Fluorescein and its derivative FITC, common stains for proteins and other biomolecules, have been selected, so that these

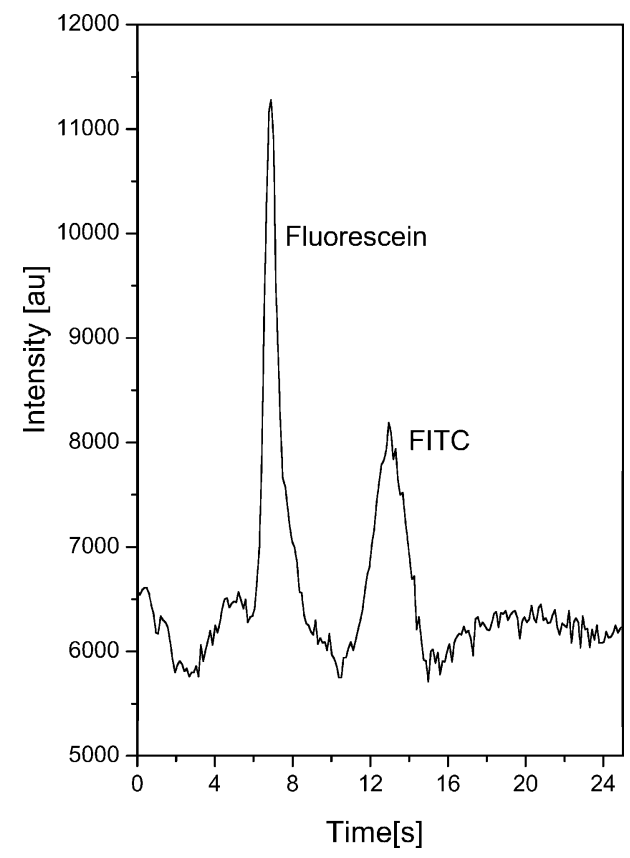

Fig. 2. Electropherogram for the separation of a $5 \mathrm{nM}$ mixture of fluorescein and FITC. The two peaks are baseline separated. Separation electric field was $1500 \mathrm{~V} / \mathrm{cm}$ and separation distance was $13 \mathrm{~mm}$ (laser power $5 \mathrm{~mW}$ ).

studies should potentially be transferable to labeled biomolecules. A simple cross of two microchannels is used in this work. In the loading phase, the sample is pumped from the sample reservoir through the cross injector. In the separation phase, the analyte in the cross injector is dispensed in the separation channel and separated according to the different mobilities of the molecules. Floating or pinched injection methods have been used in this work (see Fig. 1).

The separation efficiency in the PDMS channels was studied with the dyes fluorescein and FITC. Fig. 2 shows the separation of a $5 \mathrm{nM}$ solution of these two dyes at a separation voltage of $1500 \mathrm{~V} / \mathrm{cm}$. The two peaks are baseline separated and allow to calculate the number of theoretical plates, $N$, according to (Jorgenson and Lukacs, 1981)

$N=5.54\left(\frac{t}{w}\right)^{2}$

where $w$ denotes the full peak width at the half maximum and $t$ the migration time, respectively. $N$ resulted in 349 for fluorescein and 375 for FITC. 


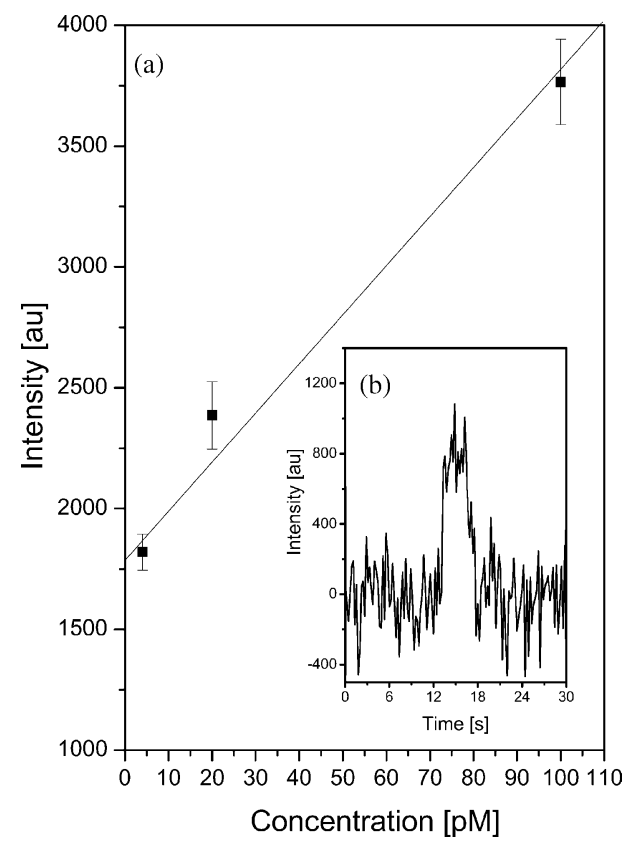

Fig. 3. (a) Peak heights for fluorescein injections from 4 to $100 \mathrm{pM}$. Error bars indicate standard deviation from three injections. The line represents a linear fit of data with $r=0.989$. (b) Electropherogram for the LIF detection of $100 \mathrm{fM}$ fluorescein injected into a PDMS device. The original data were baseline corrected and subtracted from background. Laser power was $10 \mathrm{~mW}$ for (a) and $25 \mathrm{~mW}$ for (b), respectively.

With $13 \mathrm{~mm}$ separation distance 37.2 and $34.7 \mu \mathrm{m}$ for the height equivalent of a theoretical plate $H,{ }^{1}$ for fluorescein and FITC, respectively, are achieved. This is only slightly elevated to separation data reported by Zhang and Manz (2001) in PDMS microfluidic systems with a conventional cross injector and the floating injection method. The resolution $R$ (Jorgenson and Lukacs, 1981) of the two fluorescent dyes resulted in 2.92 , which is also in good agreement with recently published data for the separation of fluorescein and FITC by Zhang and Manz (2001), representing excellent separation efficiency in PDMS devices.

Injections of fluorescein solutions demonstrated a linear dependence ranging from 4 to $100 \mathrm{pM}$ (see Fig. 3). In glass chips, detection limits of fluorescein with LIF detection of $1 \mathrm{pM}$ (Ocvirk et al., 1998) and $30 \mathrm{pM}$ (Chiem and Harrison, 1997) have been

\footnotetext{
${ }^{1} H$ is the separation distance divided by $N$.
}

reported. Thus, these results represent excellent detection performance of PDMS competitive with glass devices, whereas a signal to noise $(\mathrm{S} / \mathrm{N})$ ratio of 17 was obtained for the injections of $4 \mathrm{pM}$. Furthermore, the detection of a $100 \mathrm{fM}$ fluorescein solution could be achieved as demonstrated in the electropherogram of Fig. 3 (inset). This is to our knowledge the smallest dye concentration ever injected and detected in a microchip. With respect to the detection volume defined by the pinhole and the channel dimensions, roughly 10-100 molecules have been detected. Although Ocvirk et al. (1998) report on $300 \mathrm{fM}$ detection limit for fluorescein on a microchip, those data were obtained under continuous flow conditions and not with an injected sample.

\subsection{Protein detection}

The detection of fluorescein-labeled avidin was further investigated in BSA coated PDMS microchannels. Fig. 4 demonstrates the electropherogram obtained for the injection of $84 \mathrm{nM}$ avidin at a separation voltage

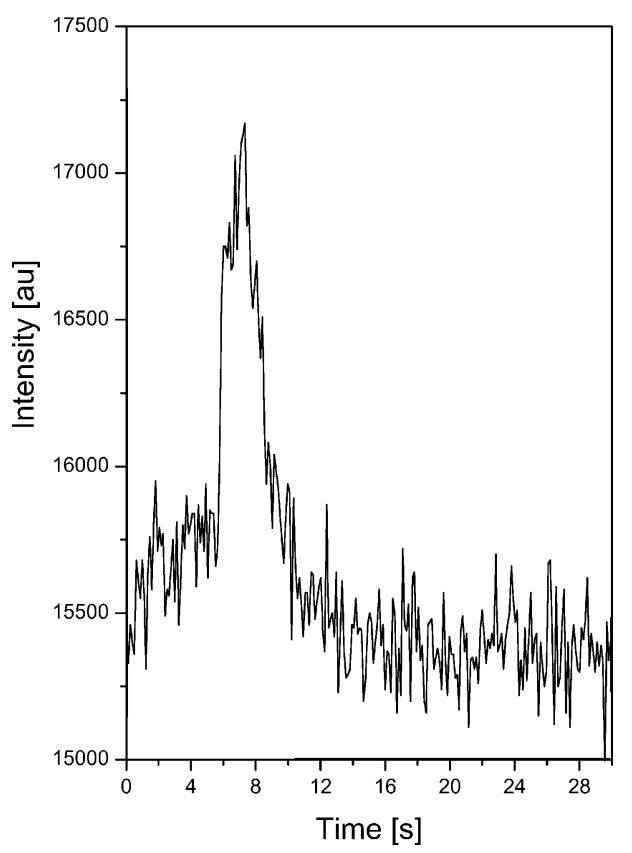

Fig. 4. Electropherogram for the injection of $84 \mathrm{nM}$ fluorescein-labeled avidin. (Electrical field strength during separation was $1500 \mathrm{~V} / \mathrm{cm}$, separation distance $6 \mathrm{~mm}$, floating injection, laser power $25 \mathrm{~mW}$ ). 
of $1500 \mathrm{~V} / \mathrm{cm}$ and a separation length of $6 \mathrm{~mm}$. The signal to noise ratio obtained for this protein peak was 10 , so that with a linear extrapolation to a $\mathrm{S} / \mathrm{N}$ ratio of 3, a theoretical detection limit of $25 \mathrm{nM}$ avidin can be expected. This is in excellent agreement with previous detection limits of proteins reported by microchip CE on glass chips (Liu et al., 2000b). The height equivalent of a theoretical plate for this avidin injection is calculated as $222 \mu \mathrm{m}$, indicating a lower separation efficiency compared to the evaluated dyes, which can be attributed to an absorptive interaction of avidin with the PDMS surface with the consequence of a reduced separation efficiency. Low separation efficiencies of proteins and peptides in PDMS have been reported by others due to the hydrophobic properties of the PDMS surface (Lacher et al., 2003).

\subsection{DNA injection and separation}

Recently, we have demonstrated an alternative method to gel based DNA separations in microchips (Duong et al., 2003). Instead of diluted polymer solutions or cross-linked gels introduced into microdevices, structured microchannels were proposed to achieve size-dependent DNA mobility in free solution. This could be demonstrated for $\lambda$ - and T2-DNA in channels with periodic structuring of the microchannel walls (Duong et al., 2003). The separation of DNA molecules according to their electrophoretic behavior in these structured microchannels relies on a controlled and reproducible injection. For this reason, we carried out sensitive video microscopy studies of the injection process with fluorescently stained DNA in a microdevice with a cross injector. Fig. 5 demonstrates a sequence of the pinched injection for DNA sampling. A $60 \mathrm{pM}$ DNA solution was pumped from the sample reservoir through the cross injector (Fig. 5a(i)). In order to avoid analyte leakage into the separation channel, voltages were applied to the buffer and waste reservoir in order to confine the sample in the crossing. For the injection into the separation channel, the electric field was switched and DNA was transported due to the electrophoretic flow in the separation channel (Fig. 5a(ii,iii)). With the pinched injection, usually around 10 molecules could be injected in $0.1 \mathrm{fL}$ injection volume under these conditions.

With a lower concentration $(6 \mathrm{pM})$ and a smaller electric field in the injection phase $(+150$ and $+75 \mathrm{~V}$ at channels 3 and 4, respectively), we were able to inject a single DNA molecule, which is demonstrated in Fig. 5b. With this injection method, it should be possible to carry out single DNA molecule investigations in microdevices. The single DNA molecule can be transported by electrokinetic forces to an area, where a single molecule investigation can further be carried out, such as binding studies to distinct receptors.

For DNA separations, the cross injector was coupled to a microchannel with $3 \mu \mathrm{m}$ periodical structures. (a)

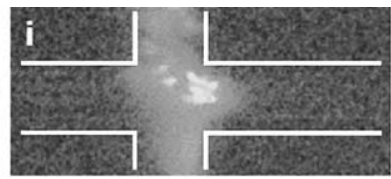

(b)

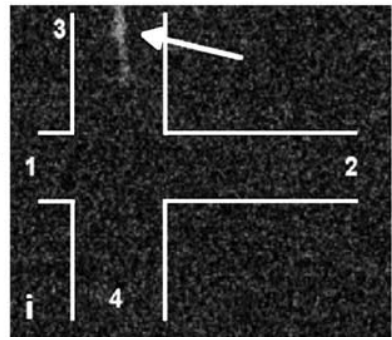

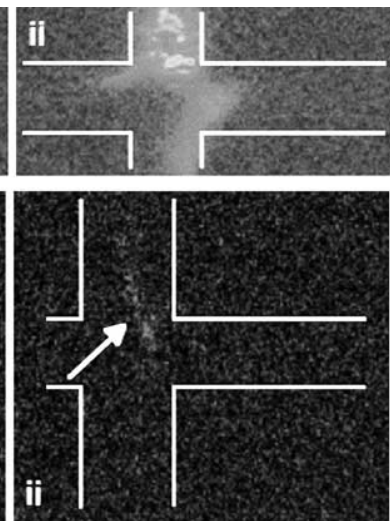
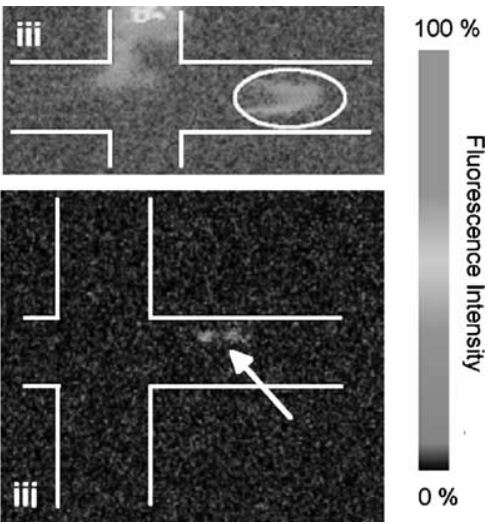

Fig. 5. (a) Injection of several DNA molecules: (i) YOYO-1 stained DNA molecules (60 pM $\lambda$ - and T2-DNA each) loading the cross injector, (ii) DNA molecules are leaving the cross injector and (iii) injected DNA molecules in the separation channel. (b) Injection of one single DNA molecule: (i) DNA before the cross injector, (ii) DNA in the cross injector and (iii) DNA injected into the separation channel. The DNA concentration was reduced to $6 \mathrm{pM}$ and lower electrical fields were used during injection (see experimental section). 


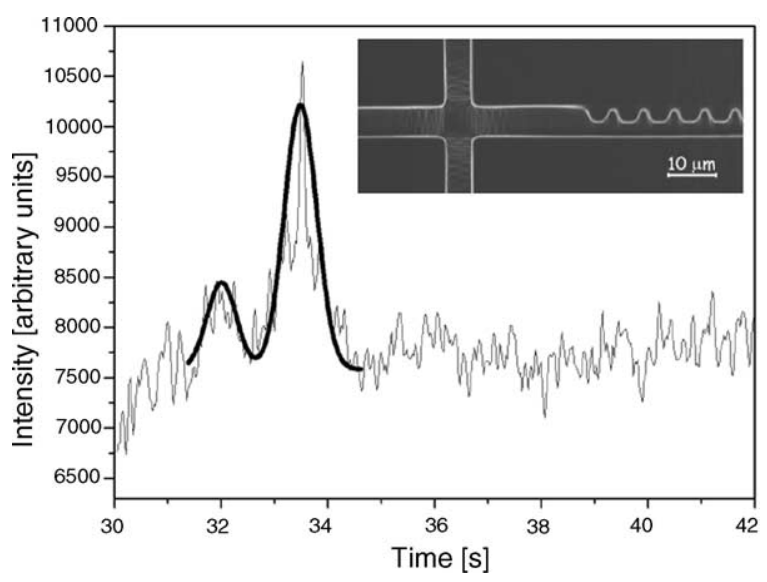

Fig. 6. Electropherogram of the successful separation of $\lambda$ - and T2-DNA (each $60 \mathrm{pM}$ ) in a microchannel with $3 \mu \mathrm{m}$ periodical structures. A CCD-camera is placed at a distance of $3 \mathrm{~mm}$ (corresponding to approximately 500 periods) from the injector (separation electrical field $50 \mathrm{~V} / \mathrm{cm}$ ) Inset: Scanning electron micrograph of a $3 \mu \mathrm{m}$ structured PDMS channel: Channels composing the cross injector are $6 \mu \mathrm{m}$ wide with linear walls. The periodical structures of the separation channel form cavities of $3 \mu \mathrm{m}$ extension. The depth of the microchannel is $2.7 \mu \mathrm{m}$.

A scanning electron micrograph shows the periodic structures of this PDMS device with the cross injector (Fig. 6, inset). Using the pinched injection method, a mixture of $\lambda$ - and T2-DNA ( $60 \mathrm{pM}$ each) was injected and separated due to the different mobilities of the two DNA species in the structured microchannel. An electropherogram of the two peaks from such a DNA separation is presented in Fig. 6. Recently, we could demonstrate that $\lambda$-DNA migrates faster than the longer T2-DNA molecule in the structured microchannels (Duong et al., 2003). This migration order is also found in Fig. 6. The first peak can be attributed to the shorter and the second to the longer T2-DNA according to the fluorescence intensity. T2-DNA contains approximately three times more fluorescent intercalant YOYO so that a higher fluorescence emission intensity is expected. Indeed, the peak heights of the two peaks yield a ratio of $1: 3$, corresponding to the ratio of YOYO intercalated in the two DNA types. Careful inspection of the video sequence of this separation additionally revealed that the second peak contains the longer T2-DNA molecules.

It is interesting to note that the observed molecular mobility within this separation is opposed to the case of entropic traps as reported by Han and Craighead
(2000, 2002). However, each time, exclusively obstacles in the dimension of the radius of gyration of the investigated DNA molecules were used, the same mobility than in our case has been reported (Inatomi et al., 2003; Kaji et al., 2003). This effect, however, not completely understood, is currently under investigation experimentally as well as by theoretical simulations.

\section{Conclusion}

In summary, we have demonstrated excellent performance of PDMS devices in respect of detection and separation efficiency of dyes and proteins, which are comparable to those obtained in glass chips. Small analyte molecules like fluorescent dyes can be separated efficiently in PDMS microdevices and $\mathrm{nM}$ protein concentrations can be detected under electrophoretic flow conditions. The PDMS microdevices served further for the detection of minute analyte concentrations down to the $\mathrm{fM}$ range with the dye fluorescein resulting in actually a sensitivity of 10-100 molecules.

Manipulation of minute DNA samples with volumes smaller than one femtoliter, containing only several molecules as well as single molecule injection in PDMS devices have been demonstrated. Additionally, with periodic channel wall structures the gel-free separation of a model DNA sample could be demonstrated. Thus, PDMS microfluidic devices can be applied for the analysis of minute analyte amounts reaching the single molecule level.

\section{Acknowledgements}

Financial support from the Deutsche Forschungsgemeinschaft in the framework of the Sonderforschungsbereich 613 (Project number D2) as well as from the microchip UV LIF project (number An 370/1-1) are gratefully acknowledged. We thank Prof. Jürgen Brugger, EPFL (Switzerland), for technical support.

\section{References}

Chabinyc, M.L., Chiu, D.T., McDonald, J.C., Stroock, A.D., Christian, J.F., Karger, A.M., Whitesides, G.M., 2001. An integrated fluorescence detection system in poly(dimethylsiloxane) for microfluidic applications. Anal. Chem. 73, 44914498 . 
Chiem, N., Harrison, D.J., 1997. Microchip-based capillary electrophoresis for immunoassays: analysis of monoclonal antibodies and theophylline. Anal. Chem. 69, 373-378.

Delamarche, E., Schmid, H., Michel, B., Biebuck, H., 1997. Stability of molded polydimethylsiloxane microstructures. Adv. Mater. 9, 741-746.

Duffy, D.C., Cooper McDonald, J., Schueller, O.J.A., Whitesides, G.M., 1998. Rapid prototyping of microfluidic systems in poly(dimethylsiloxane). Anal. Chem. 70, 4974-4984.

Duong, T., Kim, G., Ros, R., Streek, M., Schmid, F., Brugger, J., Ros, A., Anselmetti, D., 2003. Size dependent free solution DNA electrophoresis in structured microfluidic systems. Microelectron. Eng. 67-68, 905-912.

Effenhauser, C.S., Bruin, G.J.M., Paulus, A., Ehrat, M., 1997. Integrated capillary electrophoresis on flexible silicone microdevices: analysis of DNA restriction fragments and detection of single DNA molecules on microchips. Anal. Chem. 69, 3451-3457.

Han, J., Craighead, H.G., 2000. Separation of long DNA molecules in a microfabricated entropic trap array. Science 288, 10261029 .

Han, J., Craighead, H.G., 2002. Characterization and optimization of an entropic trap for DNA separation. Anal. Chem. 74, 394 401.

Inatomi, K., Izuo, S., Lee, S., Ohji, H., Shiono, S., 2003. Electrophoresis of DNA in micro-pillars fabricated in polydimethylsiloxane. Microelectron. Eng. 70, 13-18.

Jiang, G., Attiya, S., Ocvirk, G., Lee, W.-E., Harrison, D.J., 2001. Red diode laser induced fluorescence detection with a confocal microscope on a microchip for capillary electrophoresis. Biosens. Bioelectron. 14, 861-869.

Jorgenson, J.W., Lukacs, K.D., 1981. Zone electrophoresis in opentubular glass capillaries. Anal. Chem. 53, 1298-1302.

Kaji, N., Takamura, Y., Horiike, Y., Nakanishi, H., Nishimoto, T., Baba, Y., 2003. Fast separation of large DNA by nanopillar chip. Proc. $\mu$ TAS 2, 1315-1318.

Lacher, N.A., de Rooij, N.F., Verpoorte, E., Lunte, S.M., 2003. Comparison of the performance characteristics of poly(dimethylsiloxane) and pyrex microchip electrophoresis devices for peptide separations. J. Chromatogr. A 1004, 225235.

Liang, Z., Chiem, N., Ocvirk, G., Tang, T., Fluri, K., Harrison, D.J., 1996. Microfabrication of a planar absorbance and fluorescence cell for integrated capillary electrophoresis devices. Anal. Chem. 68, 1040-1046.

Liu, Y., Foote, R.S., Culbertson, C.T., Jacobson, K.B., Ramsey, R.S., Ramsey, J.M., 2000a. Electrophoretic separation of proteins on microchips. J. Microcolumn Sep. 12, 407411.

Liu, Y., Foote, R.S., Jacobson, S.C., Ramsey, R.S., Ramsey, J.M., 2000b. Electrophoretic separation of proteins on a microchip with noncovalent, postcolumn labeling. Anal. Chem. 72, 46084613.

Ng, J.M.K., Gitlin, I., Strook, A.D., Whitesides, G.M., 2002. Components for integrated poly(dimethylsiloxane) microfluidic systems. Electrophoresis 23, 3461-3473.

Ocvirk, G., Munroe, M., Tang, T., Oleschuk, R., Westra, K., Harrison, D.J., 2000. Electrokinetic control of fluid flow in native poly(dimethylsiloxane) capillary electrophoresis devices. Electrophoresis 21, 107-115.

Ocvirk, G., Tang, T., Harrison, D.J., 1998. Optimization of confocal epifluorescence microscopy for microchip-based miniaturized total analysis systems. Analyst 123, 1429-1434.

Slentz, B.E., Penner, N.A., Lugowska, E., Regnier, F.E., 2001. Nanoliter capillary electrochromatography columns based on collocated monolithic support structures molded in poly(dimethyl siloxane). Electrophoresis 22, 3736-3743.

Slentz, B.E., Penner, N.A., Regnier, F.E., 2003. Protein proteolysis and the multi-dimensional electrochromatographic separation of histidine-containing peptide fragments on a chip. J. Chromatogr. A 984, 97-107.

Xia, Y., Whitesides, G.M., 1998. Softlithographie. Angewandte Chemie 110, 568-594.

Zhang, C.-X., Manz, A., 2001. Narrow sample channel injectors for capillary electrophoresis on microchips. Anal. Chem. 73, 2656-2662. 